\title{
Belphégor
}

\section{J. Andrew Deman, The Margins of Comics: The Construction of Women, Minorities, and the Geek in Graphic Narrative}

\section{Chris Reyns-Chikuma}

\section{(2) OpenEdition \\ Journals}

Electronic version

URL: http://journals.openedition.org/belphegor/1035

DOI: $10.4000 /$ belphegor.1035

ISSN: 1499-7185

Publisher

LPCM

\section{Electronic reference}

Chris Reyns-Chikuma, « J. Andrew Deman, The Margins of Comics: The Construction of Women, Minorities, and the Geek in Graphic Narrative », Belphégor [Online], 15-2 | 2017, Online since 16

November 2017, connection on 24 September 2020. URL : http://journals.openedition.org/belphegor/ 1035 ; DOI : https://doi.org/10.4000/belphegor.1035

This text was automatically generated on 24 September 2020 .

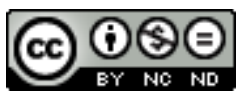

Belphégor est mis à disposition selon les termes de la Licence Creative Commons Attribution - Pas d'Utilisation Commerciale - Pas de Modification 4.0 International. 


\section{J. Andrew Deman, The Margins of Comics: The Construction of Women, Minorities, and the Geek in Graphic Narrative}

Chris Reyns-Chikuma

\section{REFERENCES}

J. Andrew Deman, The Margins of Comics: The Construction of Women, Minorities, and the Geek in Graphic Narrative. Nuada Press, 2015. 


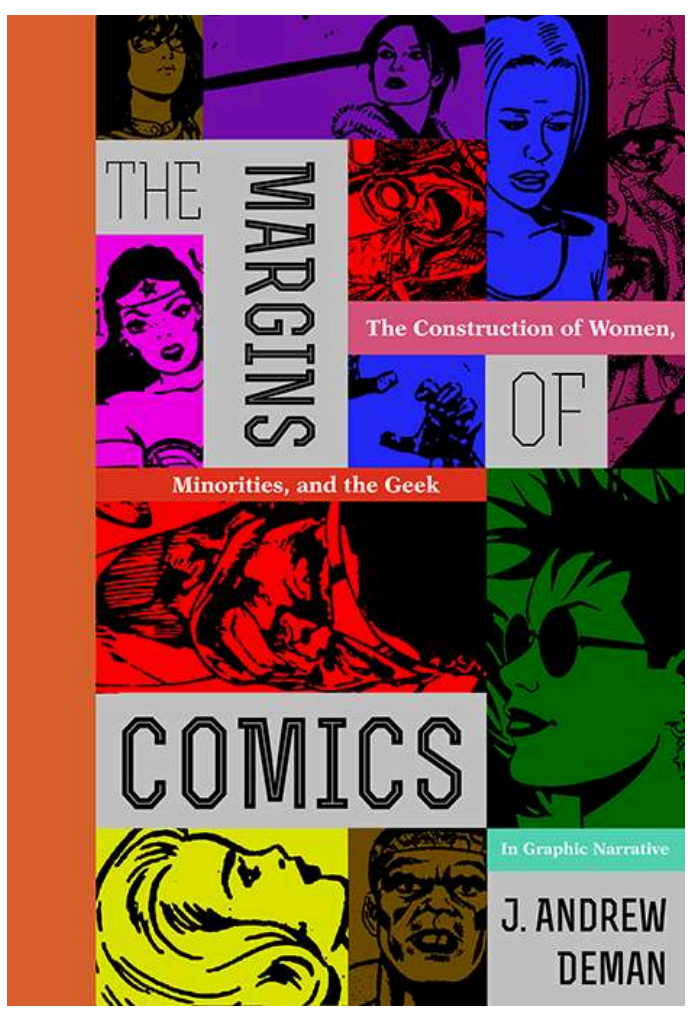

1 Comme l'explique Deman dans son introduction, il y a de nombreuses raisons qui font que les comics ont mis si longtemps pour être reconnus comme un art aux USA et au Canada (Deman est canadien et enseigne à l'université de Waterloo, en Ontario). Il cite alors plusieurs facteurs donnés par divers auteurs. Parmi ceux-ci: le format, puisque les comics étaient jetables (Sabin); la popularité souvent présentée comme équivalente à la vulgarité, et l'escapisme (Groensteen); la crainte que l'image puisse supplanter le texte et la résistance à la visualité dans le discours littéraire dominant (Mitchell); les exigences économiques, de type industriel, placées sur les artistes pour cet art déjà extrêmement exigeant en temps (Wolk); et la négligence des médias et de ses genres (surtout le genre « superhéros ») par les universitaires (Scholz).

2 A tous ces facteurs, Deman ajoute les pratiques du "othering", que l'on pourrait traduire par "aliéner" au sens de rendre quelqu'un/e radicalement autre, ou par un néologisme comme l'" autracisation» (« ostracisation» de l'autre). Par ces pratiques, les comics s'aliènent une partie du public, comme les minorités visibles qui ne se reconnaissent pas dans ces publications ainsi que les minorités éduquées progressistes qui sont souvent les gardiennes des temples culturels et qui n'acceptent pas ces discriminations. Pour ce concept, Deman s'inspire principalement d'Edward Saïd, qui, dans Orientalism, a montré comment la littérature peut contribuer à créer des perceptions imaginaires du soi et de l'autre qui impliquent une hiérarchie où l'autre est évidemment inférieur. Saïd permet toutefois une agentivité [agency] de l'auteur (surtout littéraire; cela est moins évident dans d'autres médias) pour produire une œuvre qui va à l'encontre des impératifs culturels dominants. Ce sont ces nouvelles pratiques subversives qui sont de plus en plus courantes dans le mouvement "comicsas-literature", qui permettent aux comics d'être reconnus comme art depuis 20-30 ans. ${ }^{1}$

$3 \mathrm{Si}$, comme la bibliographie de l'ouvrage l'indique, il y a des prédécesseurs à la critique de ces pratiques discriminatoires dans le domaine de la représentation dans les comics 
(on citera entre autres pour les femmes, Trina Robbins, It Ain't Me Babe, 1970, et pour les minorités en particulier noires aux USA, Jan Pieterse, White on Black, 1992), Deman reste innovateur et convaincant dans cet ouvrage de par sa méthodologie et ses exemples.

4 Deman utilise les notions barthésiennes de dénotation (littérale) et connotation (symbolique) développées dans «Rhétorique de l'image » dès 1964 et synthétisées par John Fiske dans la formule « la dénotation est ce qui est photographié, la connotation est comment c'est photographié » (cité p.15). Barthes continue en écrivant que "l'image dénotée naturalise le message symbolique, elle innocente l'artifice sémantique de connotation » (cité p. 17).

5 Mais les caractéristiques spécifiques des comics rendent ce processus de naturalisation plus fort que dans le cas de la publicité envisagé par Barthes, car les comics utilisent le dessin stylisé et en particulier la caricature comme base, offrant à l'artiste une plus grande capacité de créer des messages symboliques. En effet il n'y a pas de dessin sans style (Barthes, cité p. 18). Par exemple une femme hypersexualisée dénote une femme et donc naturalise par là l'interprétation sexiste de l'hypersexualisation.

6 L'un des problèmes essentiels pour les comics (comme pour la BD) est son rapport avec la caricature. Celle-ci, par sa nature déformante mais aussi vu le contexte nationaliste dans lequel elle s'est épanouie, a souvent été assez "autracisante». Les comics sont aussi eux-mêmes marqués par une série de préjugés autracisants et le lecteur n'est donc pas guidé dans son jugement par ce que ce média peut faire mais plutôt par ce qu'il a fait (Hatfield, cité p. 12).

$7 \quad$ Les artistes doivent donc constamment montrer qu'ils/elles sont conscient/e/s de ces problèmes autracisants. Comme Deman le prouve c'est en entrant en dialogue avec ce passé que les auteur/e/s de ces nouveaux comics ont pu réinterpréter et déconstruire ces tendances autracisantes des comics, profondément ancrées dans l'histoire et la sémiotique de ce média (p. 121).

8 Les artistes contemporains ont alors cherché à utiliser les techniques des comics pour contrer ces connotations [in]volontairement discriminatoires. Satrapi (la seule auteure francophone citée dans cette étude mais qui occupe une position centrale pour les comics et la BD étant donné son succès mondial et le rôle de l'Iran/l'Islam dans le monde aujourd'hui) est un bon exemple puisqu'elle a utilisé les caractéristiques minimales pour déconstruire des stéréotypes, même si contrairement à l'optimisme que semble montrer Deman, le résultat est nécessairement limité par les forces extérieures qui constituent l'arrière-fond de réception de l'œuvre.

Le livre est simplement et clairement divisé en 3 chapitres plus ou moins égaux; le premier s'intéresse aux représentations du genre (gender), le deuxième celles des minorités visibles, et le troisième, celles $\mathrm{du}$ " geek ». Le tout est suivi d'une très brève conclusion (168-70).

10 Le premier chapitre (25-80) qui s'intitule «L'impératif sexuel: représentations du genre dans la forme des comics» est le plus long (pp. 25-80=55p.). L'attente que toute personne se fait d'une héroïne de comics est le résultat de multiples exemples répétés du même modèle décrit par Wolk «femmes à la poitrine généreuse portant un soutif, un string et des hauts talons et qui se contorsionnent dans des positions impossibles " (ma traduction; cité p. 25). On le sait, les comic books ont longtemps visé un public jeune et masculin. Conséquemment, ils ont longtemps créé une vision idéalisée des femmes que Deman appelle «the feminine comics» (p. 26). Dépasser celle-ci est un 
objectif clé du mouvement des comics-comme-littérature. L'auteur se concentre d'abord sur Wonder Woman pour ensuite relire The Maxx de Sam Kieth et «Bomb Scare » de Adrian Tomine comme entreprises révisionnistes. Dans un troisième temps, Deman donne évidemment la parole aux comics faits par des femmes comme ceux de Phoebe Gloeckner, qui, dans A Child's Life and Other Stories [une vie d'enfant et autres histoires], a produit un comics/roman graphique sur le sexe très explicite mais antisexualisant.

11 Si les comics sont souvent critiqués pour leur misogynie, cela n'a pas toujours été le cas. Ainsi Perry a montré le rôle crucial que la caricature a joué en faveur du vote des femmes au début du siècle; d'autre part, jusqu'aux années 1950, il existait de nombreux comics pour femmes ou au moins pour femmes aussi. Le Comics Code Authority de 1954 a certainement joué un rôle négatif en faisant des comics un genre de pur divertissement évitant toute controverse; il a eu pour conséquence entre autres de rendre impossible la continuation de "romances », genre pour femmes (même s'il était souvent créé par des hommes-ce que Deman aurait dû souligner puisque ces romances n'étaient pas non plus nécessairement des modèles à suivre pour des femmes modernes). De même, en réaction contre les nombreux interdits du CCA, l'underground, dominé presque exclusivement par des hommes, considérait qu'une image de femme sexualisée était révolutionnaire et donc a complètement échoué à produire une alternative aux conventions sexistes des comics. (p.27 et pp. 52-53)

Deman se tourne alors vers le texte fondamental de Laura Mulvey, qui étudie le sexisme dans la culture visuelle à travers la notion maintenant célèbre, en particulier dans les études filmiques, de "scopophilie", tout en rejetant cependant ses aspects problématiques comme la peur de la castration (p.28). Les nombreuses et récentes études sur Wonder Woman (héroïne créée en 1940 principalement par un homme, William M. Marston) confirment l'interprétation de Deman selon laquelle Wonder Woman est sexualisée de diverses manières (costume, tenue, position, torture, et obsession du "bondage ») déconstruisant l'agenda féministe explicite de l'auteur. Il conclut alors que "l'empowerment [capacitation/empouvoir] narratif des personnages féminins est déconstruit par la représentation visuelle [sexualisante] » (40).

Maxx (56-63) a aussi été créé par deux hommes; mais en utilisant un style expérimental, ce comix défie les stéréotypes et conventions des comics, et en particulier des "feminine comics", à travers trois personnages: Julie, qui après s'être fait violer, abandonne ses études pour devenir travailleuse sociale indépendante; Maxx, un sans abri qui malgré son physique de super-héros, ne se comporte pas comme tel; et Gone, le super-méchant, violeur en série. Julie n'est pas seulement présentée comme une femme qui agit comme un "homme», mais comme un être multidimensionnel qui est capable de jouer plusieurs rôles sociaux.

Après avoir donné un autre exemple de comics créé par un auteur (Adrian Tomine, p.64-70), rien de plus important que de terminer ce chapitre par des exemples de comics faits par des femmes (71-80). La prise de parole par des femmes s'est faite en 1970 à travers deux anthologies, l'une, It aint me babe de Trina Robbins, plus politique, et l'autre plus autobiographique, Twisted Sisters de Aline Kominsky-Crumb. Cette dernière, lente d'abord à connaître le succès, aura une influence à plus longue échéance. L'œuvre de Gloeckner en est témoin. Elle est très proche des œuvres de Keith et Tomine mais sur un mode plus confessionnel à l'intérieur d'un fort mouvement d'œuvres « autographiques » rassemblant Spiegelman, Satrapi, Bechdel, .... Elle utilise 
efficacement la multimodalité typique des comics et des techniques comme l'acteimage (voir Kress \& Van Leeuwen, inspiré du «speech act » de Austin) comme dans le cas où on voit l'auteur-adulte qui regarde directement le lecteur, comme s'il exigeait une réponse de lui. Elle représente la voix d'une femme forte dans un monde dominé par les hommes racontant des histoires sexuellement explicites mais nullement érotiques, puisque le personnage féminin est abusé et pris comme objet sexuel tour à tour par le père, l'ami, et le petit ami.

15 Le deuxième chapitre s'intitule «minorités visibles: représentation de la race dans la forme des comics » (80-122=42p.). Il contient 9 exemples dont les premiers sont discutés pour montrer leurs limites et les plus contemporains pour montrer leurs qualités. Dans les premiers comics des années 1900, comme par exemple ceux d'un McCay, Deman montre les rapports avec la caricature et le racisme de cette première longue période, où les stéréotypes raciaux sont repris sans trop y penser, tout en créant une forme artistique déjà très élaborée. Il saute ensuite directement aux années 60-70 à l'époque des luttes des droits civils, avec des superhéros comme Luke Cage, Black Panther, et Storm (p. 99) pour montrer les limites de ces représentations souvent encore créées par des auteurs blancs. Il passe enfin davantage de temps sur l'époque contemporaine avec Katchor (109-112) où le côté performatif de la notion de race ou d'ethnie est démontré entre autre à travers la mise en images des personnages au théâtre; puis Satrapi (112-113, discutée plus haut); Sacco, qui utilise la caricature mais contre l'auteur luimême, qui est aussi mis en image, et non contre les autres (113-114); et enfin, Spiegelman, qui dans son Maus met en scène entre autres le masque pour déconstruire la notion de race (116-122).

16 Le troisième chapitre s'intitule « Un déguisement vaporeux [squinkie]; représentation du geek dans la forme des comics (123-67). Le sujet de ce chapitre sur les "geeks» autracisés est sans doute le plus original. Il est aussi peut-être le moins applicable en France dans la mesure où la BD ne semble pas avoir produit ce genre de concept et de réalité comme aux USA (ou au Japon avec l'otaku). Mais paradoxalement, même aux USA, les geeks, qui sont pourtant des marginaux, ont rarement été présentés comme des parias dans les comics; au contraire, ceux-ci ont plutôt produit des messages qui parlent aux geeks, quitte à les intégrer à ces messages "autracisants ", racistes et sexistes, car le geek a très souvent été perçu comme un adolescent ou jeune adulte mâle et blanc.

17 Deman décrit alors trois périodes: celle des premiers superhéros, comme Superman, qui apparait comme l'exemple typique du fantasme de pouvoir sexuel et social (125-48=23p.); puis celle de l'ère Marvel, c'est-à-dire des années 60 (celle aussi des premiers superhéros noirs) surtout avec Spiderman, qui tendait à présenter des parallèles entre le geek et les minorités raciales ostracisées (148-153=5p.); et enfin l'époque contemporaine avec l'exemple de Jimmy Corrigan, geek que Chris Ware présente avec un sens de pathos tragique (154-167=13p.).

18 S'appuyant sur divers chercheurs comme Lori Kendall et Ron Eglash, le critique affirme que le parallèle entre les trois types de discrimination est parfaitement valable car elles sont toutes trois des constructions qui donnent le pouvoir à deux formes de racisme, contre les noirs vus comme hypersexuels et hypermasculins, et contre les Asiatiques comme hyposexuels et hypomasculins. Conséquemment ce parallèle implique que les blancs sont perçus "comme le parfait équilibre entre ces deux extrêmes» (p. 125). Plutôt qu'un inadapté social obsédé par une passion (les livres, les nouvelles 
technologies, ...), le geek est donc simplement quelqu'un qui ne performe pas bien sa masculinité (125).

Superman est présenté comme fantasme de geek tour à tour à travers son déguisement de journaliste, son amour pour Loïs Lane, et son ennemi, Lux Luthor. Superman est en effet forcé de se déguiser en journaliste de seconde zone, ignoré et même harassé par son patron, ses collègues et celle dont il est amoureux. Ses lunettes sont le symbole de " geekité » comme la cape et les collants sont ceux du superhéros. Clark Kent a besoin de rester journaliste car cela lui donne des informations dont il a besoin pour agir comme superhéros. Il doit donc accepter ce déguisement de geek et les souffrances qui vont avec. Deman conclut que «le message est simple: il y a de la noblesse dans la souffrance du geek» (130). Outre l'abondance de documents biographiques qui présentent les deux auteurs de Superman (Siegel \& Shuster) comme des geeks, l'importance du geek dans cette série apparaît le plus clairement dans deux faits représentés symboliquement. D'abord, la femme aimée refusant le geek est en fait présentée comme pas spécialement intelligente, puisque contrairement au lecteur (ado ou jeune adulte) elle ne comprend pas que Superman et Clark sont la même personne. Ensuite, de nombreuses scènes sont plus que suggestives sur le plan sexuel. Ainsi comme Deman le montre, la scène de la dame en détresse rattrapée en vol par superman, répétée des dizaines de fois, peut aisément être interprétée comme une scène pré-sexuelle semblable par exemple au jeune marié qui transporte sa nouvelle épouse dans la chambre nuptiale pour la consommation de l'acte, scène toujours abruptement interrompue et laissée à l'imagination du lecteur-geek-ado-jeune adulte male selon le code moral de l'époque. De même, la femme est sauvée souvent après avoir été violentée, torturée voire violée, la présentant ainsi comme punie pour son insolence vis-à-vis de Clark-journaliste-geek, et le nerd-lecteur est donc vengé pour son humiliation (symbolique), tout en punissant aussi le «méchant » qui l'a violentée (qui est l'autre partie du lecteur-geek qui a joui de cette punition-revanche). Inversement, l'hypersexualisation de la femme, plus typique encore dans les comics que dans d'autres médias, peut être comprise comme compensation pour le geek. Enfin, Elektra, l'une des rares femmes qui ose et réussit pendant longtemps à défier le male sur son terrain de combat, est transpercée à mort avec le poignard qu'elle a osé usurper (voir le Daredevil de Frank Miller). Dans les comics de ces années 40-70, la sexualité est évidemment implicite, symbolique, connotée, et Deman explique que la sexualité explicite, violente et sexiste, des dessins underground (par exemple d'un Crumb) dans les années 50-60, est l'autre côté de la même médaille (p. 144-45).

A côté de l'attitude conquérante de Lois Lane est le conflit de Superman avec Lex Luthor, qui «simultanément condamne, punit, rationalise, et valorise l'expérience geek, mettant en scène le conflit interne de l'identité geek» (128, 145-48). Ce conflit se situe entre la définition-identité du geek, fou de technologie et asocial comme c'est le cas pour Lex, et sa passion pour le superhéros.

21 Présenté comme sans superpouvoir et typiquement comme un cadre supérieur qui commande à des hommes de main à travers des technologies (micros, écrans), Lex est l'archétype du savant-fou ou d'un super-geek opposé à Superman qui, lui, reste très humain en dépit de sa superpuissance. Les deux représentent les deux faces du geek, mais où l'une doit être finalement punie (Lex) et l'autre récompensée (Superman). « Ces comics parlent donc clairement au public geek à travers des messages et connotations qui donnent à l'imagination du lecteur-geek des éléments clés dont le geek manque en 
réalité: pouvoir physique, expérience sexuelle, et valorisation de l'identité geek » (ma traduction, p. 149).

Dans les années 60, Marvel s'adressa plus directement aux geeks en les intégrant à l'histoire avec les Spiderman, Dr. Strange, Cyclops, Mr. Fantastic, et Bruce Banner. Spiderman est en effet vraiment un geek qui, contrairement à Superman, ne doit pas prétendre en être un. De plus, Marvel, surtout Stan Lee, crée des connections directes avec les geeks à travers un courrier des lecteurs, puis un fan club qu'il appelle les "Merry Marvel Marching Society », établissant ainsi un parallèle entre le mouvement des geeks et les marches-manifestations des mouvements sociaux des droits civiques (151). Ce parallèle est le plus évident dans les comics X-Men où les mutants sont ostracisés mais où les «bons » mutants de Xavier (Martin Luther King) choisissent la voie « légale » contre la voie violente de Magneto (Malcolm x).

Viennent après les années 60 , quand le geek est donc devenu plus sympathique. Et c'est sur cette sympathie que la génération suivante va bâtir des romans graphiques qui, ayant plus de 30 pages (comic books), peuvent créer des personnages et offrir des situations beaucoup plus complexes encore. Ce nouveau format permet alors de créer plus d'empathie pour ce geek, présenté de manière à la fois héroïque et tragique (154). L'exemple le plus connu est évidemment Jimmy Corrigan. Bien que sans lunettes, le geek de Chris Ware est présenté comme typique: timide, posture bossue, petits yeux, vêtements d'enfant. Toutefois, il n'a même pas les compensations des héros de Marvel: corps musclé et superbe petite amie. Ware met en scène les fantaisies sexuelles de Jimmy lorsque par exemple il sauve une amie des bandits, la ramène à sa maison pour la soigner et commence à la déshabiller avant d'être interrompu par quelqu'un qui frappe à la porte, et il se réveille. Ware fait alors aussi la critique du mythe du superhéros sauveur comme étant une littérature escapiste, pouvant mener trop facile à la désillusion. Ainsi, Ware crée un dialogue avec l'histoire du genre superhéros, longtemps cru équivalent à tout le média "comics", pour à la fois déconstruire le mythe du superhéros, du geek, et les limites de la forme des comics.

Dans sa brève conclusion (168-170), Deman réaffirme que les artistes des comics ont une forte conscience de l'histoire de leur média et sentent qu'ils doivent faire référence au genre, par exemple celui des superhéros, pour justifier l'existence et la qualité de leur art, ce que d'autres genres/médias comme le roman ne doivent plus nécessairement faire. Ceci est en partie dû au fait que le genre/média comics inclut dès le départ le processus du othering/autracisation venant de la caricature et du dessin où les hommes apparaissent plus masculins, les femmes plus féminines, les geeks plus geeks, les noirs plus noirs, etc. Ces processus autracisants ont joué un rôle non négligeable dans la perpétuation des pratiques sociales d'autracisation (racisme, sexisme, ...). Mais ils ont aussi impacté le développement de la forme même, d'abord comme obstacle au mouvement des « comics comme littérature » et ensuite à travers le travail de révision des artistes décrits dans cette étude. Ceci montre aussi qu'en dépit du mouvement de certains chercheurs, qui tend à montrer que tous les comics se valent, il y a bien des comics qui réussissent à se distinguer du lot pour construire un genre et une société où les pratiques autracisantes sont rejetées.

Bref, après ce long compte rendu on comprendra que The Margins of Comics de J. Andrew Deman est un livre hautement recommandé! 


\section{NOTES}

1. On notera que dans Bande dessinée et littérature, paru en 2013, Jacques Durrenmatt a montré un mouvement de reconnaissance similaire pour la BD francophone mais sans aucune allusion à ces processus d'autracisation pourtant tout aussi commun dans la bande dessinée francophone.

AUTHOR

CHRIS REYNS-CHIKUMA

Université de l'Alberta 